\title{
Circumvent hesitancy between CDK4/6 and mTOR inhibitors in second-line treatment of HR+, erb2- metastatic breast cancer
}

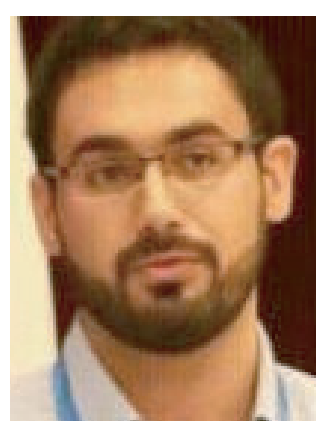

\author{
"In this era of personalized medicine, one is \\ obliged to take into consideration three \\ parameters that would constitute the outline of \\ this editorial: tumor biology, host biology and \\ patient's quality of life."
}

Elie El Rassy ${ }^{*, 1}$, Pamela Abdayem \& Joseph Kattan'

First draft submitted: 20 April 2017; Accepted for publication: 3 May 2017; Published online: 7 July 2017

Breast cancer $(\mathrm{BC})$ rates are among the highest in cancer incidence and mortality. Most commonly, these patients are erb2 negative and hormone receptor (HR)-positive and thus, receive hormonal therapy as a cornerstone of their treatment in the advanced setting [1]. The majority of these patients respond to hormonal therapy but primary or secondary resistances may be observed. Several resistance mechanisms have been shown to drive the tumor proliferation, growth, cell cycle and progression via upregulation of alternate signaling including EGFR, PI3K, mTOR and CDK4/6 pathways [2]. Thus, multiple options to overcome this resistance were developed; the most commonly recommended options to enhance hormonal therapy in the second line include the addition of either CDK4/6 or mTOR inhibitors [3]. In this era of personalized medicine, one is obliged to take into consideration three parameters that would constitute the outline of this editorial: tumor biology, host biology and patient's quality of life.

The tumor biology is the first parameter that guides the oncologist into looking in his armamentarium for possible treatment options, as it is the tumor biology that drives therapeutic outcomes. It is through understanding the resistance profile leading to progression that the CDK4/6 and mTOR pathways are eligible for inhibition. Mechanistically, mTOR inhibition decreases angiogenesis, proliferation and metabolism but also causes a cross-talk activation of CDK4/6. The inhibition of the mTOR complex 1 paradoxically activates the AKT pathway and leads to an aggressive disease after an initial response and progression-free survival (PFS) $[4,5]$. On the other hand, the CDK4/6 inhibitor inhibits the transition of the cell mitosis from G1-S by inactivating retinoblastoma $(\mathrm{Rb})$. It is noteworthy that the recent trials of CDK4/6 inhibitors excluded patients

\section{KEYWORDS}

- CDK4/6 inhibitor • metastatic breast cancer $\bullet$ mTOR inhibitors - second line 
"Choosing the best players for the all-star game remains the ultimate challenge for us oncologists." with a loss of $\mathrm{Rb}$ expression which constitutes $3 \%$ of HR-positive BC patients (NCT01536743, NCT02334527, NCT01976169). It is known that CDK4/6 is closer to the nucleus; hence, its inhibition is associated with lesser cross-talk and secondary resistance [2]. Yet, resistances were described with Rb-dependent activation of AKT and metabolic reprogramming via mTORC2 and mTORC1, respectively $[4,5]$. Moreover, while mTOR inhibitors restore endocrine sensitivity, CDK4/6 inhibitors may favor the emergence of estrogen receptor 1 (ESR1) mutations resulting in ligand-independent activity of the receptor. ESR1 mutations, detected in $20-30 \%$ of patients with metastatic ER-positive endocrine-resistant metastatic breast cancer, are a negative prognostic biomarker, responsible for acquired resistance to aromatase inhibitors [6]. In the future, we believe that the identification of such mutations by analysis of circulating tumor DNA might be used as a predictive biomarker of response to treatment with either CDK4/6 or mTOR inhibitors as well as a surveillance tool for tumor relapse [6]. Also, CDK4/6 inhibitors block cyclin D2 and cyclin D3-dependent G1-phase progression and T-cell expansion. This blockade would theoretically antagonize the action of checkpoint inhibitors, should they be used in this setting. It might also neutralize the effects of cytotoxic drugs and radiation therapy that rely on destroying cancer cells in later phases of the cell cycle.

In order to assess the host biology, the inclusion criteria in the available trials should be closely analyzed. For this matter, we discuss the pivotal trials PALOMA 3 (NCT01942135) and BOLERO 2 (NCT00863655) with particular attention to the patients' characteristics and treatment effect of the intervention drugs [7,8]. Both studies have a similar prospective design with a 2:1 randomization to Fulvestrant $(500 \mathrm{mg}$ im. q.4 weeks) with or without Palbociclib (125 mg q.d.; 3 weeks on/1 week off) in the PALOMA 3 trial and Exemestane $(25 \mathrm{mg} /$ day) with or without Everolimus $(10 \mathrm{mg} /$ day $)$ in the BOLERO 2 trial. Eligibility required HR-positive advanced $\mathrm{BC}$ that has progressed on prior endocrine therapy. The characteristics of both populations were similar for age, performance status, visceral disease and number of metastatic sites. However, PALOMA 3 extended the inclusion criteria to pre-, peri- and postmenopausal women. BOLERO 2 on the other hand had longer diseases-free survival with earlier treatments and also had a more heavily pretreated population.
In regards to efficacy, a PFS gain is observed with both drugs where Palbocilib increased PFS from 3.8 to 9.2 months (HR: 0.42 ; $95 \% \mathrm{CI}$ : $0.32-0.56 ; \mathrm{p}<0.001$ ) and Everolimus from 3.2 to 7.8 months (HR: 0.43 ; 95\% CI: $0.35-0.54$; $\mathrm{p}<0.001)$. Overall survival (OS) is not statistically significant between the two treatment arms in the BOLERO 2 trial and is not yet mature in the PALOMA 3 trial. One would attribute the lack of OS benefit to the study design and end points that were identical in both trials: the lack of power and the imbalance in poststudy salvage chemotherapy. Conversely, PALOMA 3 overcomes a major limiting factor and promises a potential OS gain with Palbociclib in view of its mechanism of action as previously described.

The patient's quality of life is undoubtedly a major parameter that physicians commonly overlook although, it should be highly considered in personalized medicine. The quality of life of the patients has been reported in both the PALOMA 3 and BOLERO 2 trials where it was ameliorated significantly only in the first trial $[7,8]$. Patients receiving everolimus suffered from adverse events (AE) including stomatitis (59\%), rash (39\%) and fatigue $(37 \%)$ [8]; in the largest cohort of patients receiving Everolimus, grade III/IV AE occurred in $42.7 \%$, treatment discontinuation in $17.1 \%$ and dose reduction in $29.6 \%$ [9]. However, in a Phase I study of Everolimus, the dose reductions of Everolimus were associated with a possible risk of losing the dose-dependent minimal inhibitory concentration of the mTOR pathway effectors (pS6 and peIF-4G) [10]. Palbociclib, on the other hand, is administered with complete dose intensity in $92 \%$ of patients and treatment discontinuation limited to $2.6 \%$. Neutropenia is the mostly deranging AE of Palbociclib, with grade III neutropenia occurring in $50 \%$ of patients and febrile neutropenia in $0.6 \%$ [7]. The prior arguments would definitely favor the use of Palbociclib over Everolimus; however, real-life studies that were published after BOLERO 2 such as the BRAWO study showed a decreased incidence of grade III/IV stomatitis (2-11\% of cases), with the majority of events occurring within the first 8 weeks of treatment and a median duration of 28 days [11].

Targeting mTOR is not yet fully valid biologically. Temsirolimus in the HORIZON trial (NCT00083993) had major AE with PFS advantage although, there is scepticism about the methods in this trial [12]. As for targeting CDK4/6 with second-generation drugs such 
as Abemaciclib and Ribociclib, we have major biological advantages. These drugs have higher potency, wider spectrum of CDK inhibition, higher blood-brain barrier absorption and less bone marrow toxicity [13,14].

In conclusion, all the options presented in this editorial are valid in the absence of a headto-head trial and safe if used properly. The art remains in choosing the appropriate sequence of these agents based on patient and disease characteristics as well as patient preferences. In the future, several developed 'tools' may help us select the optimal sequence in HR-positive metastatic BC:

- Gene expression assays, currently used in the adjuvant setting, might identify the subset of patients with long durable disease control: they would receive monotherapy in first line and combination therapies in later lines.

- Predictive biomarkers currently undergoing clinical trial evaluations such as ESR1 mutations, might predict the response to either mTOR or CDK 4/6 inhibitors.

Finally, endocrine therapy in HR-positive metastatic breast cancer is drastically improving. The next generation of trials will investigate the most effective way to combine and schedule these drugs. Choosing the best players for the all-star game remains the ultimate challenge for us oncologists. The new roster includes PI3-kinase inhibitors and combination of mTOR and CDK4/6 inhibitors.

\section{Financial \& competing interests disclosure}

The authors have no relevant affliations or financial involvement with any organization or entity with a financial interest in or financial conflict with the subject matter or materials discussed in the manuscript. This includes employment, consultancies, honoraria, stock ownership or options, expert testimony, grants or patents received or pending, or royalties.

No writing assistance was utilized in the production of this manuscript.

\section{References}

1 Pritchard KI, Chia SK, Simmons C et al. Enhancing endocrine therapy combination strategies for the treatment of postmenopausal HR+/HER2- advanced breast cancer. Oncologist 22(1), 12-24 (2017).

2 Arpino G, Wiechmann L, Osborne CK, Schiff R. Crosstalk between the estrogen receptor and the HER tyrosine kinase receptor family: molecular mechanism and clinical implications for endocrine therapy resistance. Endocr. Rev. 29(2), 217-233 (2008).

3 Rugo HS, Rumble RB, Macrae E et al. Endocrine therapy for hormone receptorpositive metastatic breast cancer: american society of clinical oncology guideline. J. Clin. Oncol. 34(25), 3069-3103 (2016).

4 O'Sullivan CC. Overcoming endocrine resistance in hormone-receptor positive advanced breast cancer-the emerging role of CDK4/6 inhibitors. Int. J. Cancer Clin. Res. 2(4), pii:029 (2015).

5 Friend S, Royce M. The changing landscape of breast cancer: how biology drives therapy. Medicines 3(1), 2 (2016).

6 Chandarlapaty S, Chen D, He W et al. Prevalence of ESR1 mutations in cell-free
DNA and outcomes in metastatic breast cancer: a secondary analysis of the BOLERO-2 clinical trial. JAMA Oncol. 2(10), 1310-1315 (2016).

7 Turner NC, Ro J, André F et al. Palbociclib in hormone-receptor-positive advanced breast cancer. N. Engl. J. Med. 373(3), 209-219 (2015).

8 Baselga J, Campone M, Piccart M et al. Everolimus in postmenopausal hormonereceptor-positive advanced breast cancer. N. Engl. J. Med. 366(6), 520-529 (2012).

9 Jerusalem G, Mariani G, Ciruelos EM et al. Safety of everolimus plus exemestane in patients with hormone-receptor-positive, HER2-negative locally advanced or metastatic breast cancer progressing on prior non-steroidal aromatase inhibitors: primary results of a Phase IIIb, open-label, single-arm, expanded-access multicenter trial (BALLET). Ann. Oncol. 27(9), 1719-1725 (2016).

10 Tabernero J, Rojo F, Calvo E et al. Dose- and schedule-dependent inhibition of the mammalian target of rapamycin pathway with everolimus: a Phase I tumor pharmacodynamic study in patients with advanced solid tumors. J. Clin. Oncol. 26(10), 1603-1610 (2008).
11 Schütz F, Grischke EM, Decker T et al. Stomatitis in patients treated with everolimus and exemestane-results of the third interim analysis of the non-interventional trial BRAWO. Poster presented at: San Antonio Breast Cancer Symposium, San Antonio, TX, 8-12 December 2015 (P4-13-08).

12 Rugo HS, Seneviratne L, Beck JT et al. Prevention of everolimus-related stomatitis in women with hormone receptor-positive, HER2-negative metastatic breast cancer using dexamethasone mouthwash (SWISH): a single-arm, Phase 2 trial. Lancet Oncol. 18(5), 654-662 (2017).

13 Wolff AC, Lazar AA, Bondarenko I et al. Randomized Phase III placebo-controlled trial of letrozole plus oral temsirolimus as first-line endocrine therapy in postmenopausal women with locally advanced or metastatic breast cancer. J. Clin. Oncol. 31(2), 195-202 (2013).

14 Hortobagyi GN, Stemmer SM, Burris HA et al. Ribociclib as first-line therapy for HR-positive, advanced breast cancer. N. Engl. J. Med. 375(18), 1738-1748 (2016). 